\title{
CANDIDA SEROLOGY IN OPEN-HEART SURGERY
}

\author{
E. R. PARSONS AND E. NASSAU \\ Department of Pathology, Harefield Hospital, Harefield, Middlesex
}

CANDIDA infection, particularly endocarditis, is a serious and often fatal complication of homograft or prosthetic heart-valve replacement (Kay et al., 1968; Murray, Buckley and Turner, 1969; Record et al., 1971). Confirmation of candida endocarditis and systemic infection by culture is time-consuming, and cultures may be negative in the presence of active infection (Kay et al, 1968). Various serological tests have been proposed to assist in diagnosis. Winner (1955) found that $64 \%$ of apparently uninfected persons had agglutinating antibodies to Candida albicans, but Stallybrass (1964) suggested that precipitating antibodies to $C$. albicans are present only in systemic and not in superficial candidosis. Lehner (1966) suggested that a titre of more than 16 in the immunofluorescence (fluorescent antibody; FA) test with anti-human whole globulin conjugate, confirms clinical infection. Remington, Gaines and Gilmer (1972) found a close correlation between immunodiffusion (ID) and counterimmunoelectrophoresis (CIE) test results, but doubts concerning the specificity of the CIE test were expressed by Hellwege, Fischer and Bläker (1972). Murray et al., (1969) in a study of the serology of candida infection after open-heart surgery concluded that positive results in ID tests are not by themselves diagnostic of candida endocarditis.

Because of its simplicity and rapidity, we have used the CIE test as a routine post-operative screening procedure to detect antibodies to $C$. albicans and for the diagnosis of suspected candida endocarditis after cardiac surgery. Since many sera gave positive reactions in the absence of clinical indications of infection, and others reacted weakly or gave doubtful reactions in the test, the CIE test alone was considered to be insufficient as a screening procedure. Therefore we investigated the value of ID, FA, and agglutination (AGG) tests as screening procedures.

\section{MATERIALS AND MethodS}

Sera

These were obtained from patients who were about to undergo, or who had undergone, operations on the heart, and from control patients and staff. The pre-operative sera were collected 1-3 days before operation and the post-operative sera between 2 months and 3 years after operation. Sera were initially divided into eight categories:

1. Endocarditis: a total of 20 post-operative sera from six patients who had developed endocarditis after homograft heart-valve replacement, shown by culture to be due to Candida spp. (table I). Only in Case 2 was a pre-operative serum available for study. In Case 6 prolonged incubation of material from the homograft yielded $C$. albicans indistinguishable from later blood-culture isolates.

Received 7 Sept. 1973; accepted 28 Jan. 1974.

J. MED. MICROBIOL.-VOL. 7 (1974) 


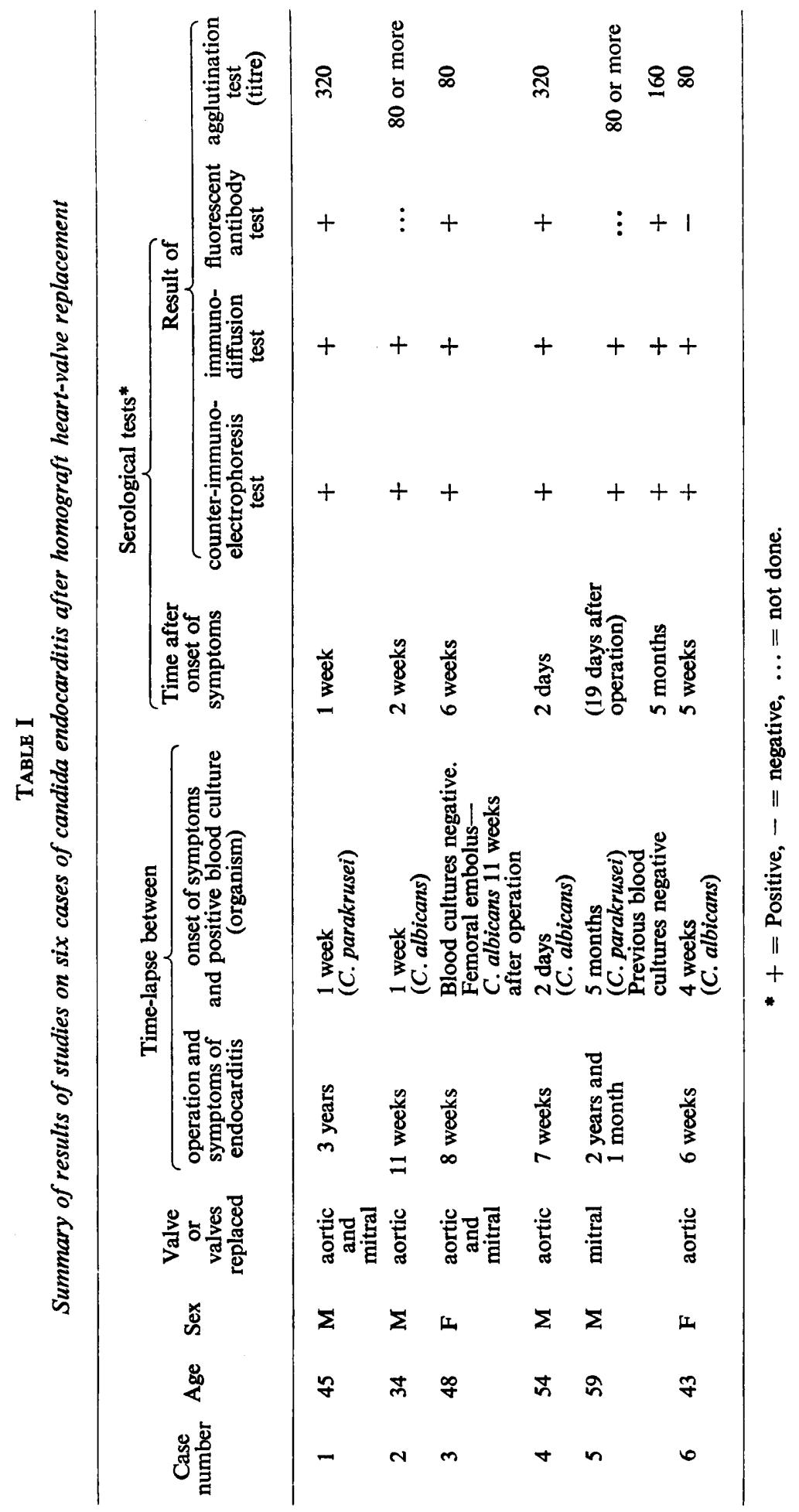


2. Candida isolated from homograft: in all six pre-operative and six-post operative sera from five patients who may have received contaminated homograft material; Candida spp. was isolated from samples of the homograft material, or of the aortic wall of the donor that had been sterilised in the same manner as the valve. In many cases Candida spp. were not isolated from the cultures of homograft or aortic-wall material until up to 3 months after the homograft had been inserted. None of these patients developed clinical endocarditis. Endocarditis Case 6, mentioned above, is not included in this group.

3. Pre-operative: 386 sera obtained 1-3 days before operation from 321 patients who subsequently underwent cardiac surgery. In 216 cases no post-operative serum has so far been examined.

4. Control: 84 sera from 84 unselected non-cardiac patients and staff, in whom superficial candidosis was not excluded.

5 and 6. Post-homograft: 278 sera from patients who had received homograft heartvalve replacements, taken from 2 months to 3 years after operation. In 38 cases a preoperative serum was also tested. This group was divided into sera obtained less than 1 year (group 5) and more than 1 year (group 6) after operation.

7 and 8. Post-cardiac surgery: 236 sera from 207 patients who had undergone cardiac surgery other than homograft heart-valve replacement. Of these patients, 88 had coronaryartery bypass-grafts, 32 mitral valvotomies, 16 atrial septal-defect repairs, 16 excisions of cardiac aneurysms, and 55 other repairs and combinations of the above procedures. In 56 cases a pre-operative serum was also examined. This group was divided into sera obtained less than 1 year (group 7) and those obtained more than 1 year (group 8) after operation.

\section{Preparation of antigens}

A preliminary study of the use of antigens prepared from various strains of $C$. albicans, $C$. parakrusei, and C. parapsilosis was made. Even in endocarditis caused by $C$. parakrusei the use of the homologous organism as antigen was found not advantageous. Subsequently, all antigens were prepared from a stock culture of $C$. albicans (no. SC100) isolated from a blood culture in 1967. This strain was found to produce better antigens than other cultures tested and conforms to the description of Type-A C. albicans (Hasenclever and Mitchell, 1961).

Mickle extract. C. albicans was grown on Sabouraud Maltose Agar (Oxoid) for 48 hours at $37^{\circ} \mathrm{C}$ in $8-\mathrm{oz}$ " medical flat" bottles. The cells were harvested and suspended to a concentration of approximately $50 \%$ packed cell volume in $0.5 \%$ phenol-saline. The suspension was then disintegrated in a Mickle shaker for 1 hour, with Ballotini beads, followed by centrifugation to remove cell debris. The supernate was titrated against strongly and weakly positive sera by immunodiffusion and counter-immunoelectrophoresis. It was found that no single dilution of antigen gave optimal precipitin lines against all sera and two dilutions of antigen were used routinely. These dilutions varied from batch to vatch and were prepared from the stock antigen by dilution in $0.5 \%$ phenol-saline and stored at $4{ }^{\circ} \mathrm{C}$.

Agglutinating suspension. C. albicans was grown on Sabouraud Maltose Agar (Oxoid) for 48 hours at $37^{\circ} \mathrm{C}$ in $8-\mathrm{oz}^{\text {" }}$ medical flats ". The cells were harvested in $0.5 \%$ formol-saline and stored at $4^{\circ} \mathrm{C}$ overnight. The suspension was then washed, resuspended in 1 in 10,000 merthiolate-saline to a concentration of $2 \%$ as determined by microhaematocrit estimation, and stored at $4^{\circ} \mathrm{C}$.

Suspension for immunofluoresence studies. C. albicans was grown on horse blood agar (Oxoid Columbia Agar Base) for 24 hours at $37^{\circ} \mathrm{C}$. This was found to give better cell dispersion and fewer hyphae than growth on Sabouraud agar or in broth. The growth was harvested in $2 \%$ phenol-saline and left at $4^{\circ} \mathrm{C}$ overnight. After washing in saline the suspension was diluted so that one loopful, when dried on a slide, would give separated cells when viewed under a $\times 20$ objective.

Multi-spot PTFE-coated $3 \times 1 \frac{1}{2}$ in. glass microscope slides (O'Neill and Johnson, 1970) were prepared, with six spots per slide. Loopfuls of the suspension were spread over each spot on the slide, air-dried and fixed in $10 \%$ methanol for $30 \mathrm{~s}$. The slides were then stored at $-20^{\circ} \mathrm{C}$. 


\section{Serological Tests}

Counter-immunoelectrophoresis test. (CIE). This was performed with a Hyland Electrophoresis Power Supply. Trays were prepared with $20 \mathrm{ml}$ of $1 \%$ Agarose (Micro-Bio Labora-

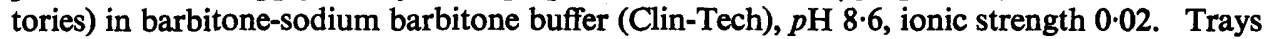
were allowed to set on a levelled surface. Thirty-two pairs of wells were cut in four columns. The well diameter was $3 \mathrm{~mm}$, with $5 \mathrm{~mm}$ between wells, centre-to-centre, and $8 \mathrm{~mm}$ between pairs of wells. The distance between columns was $11 \mathrm{~mm}$.

Undiluted serum was placed in the anodal well and antigen in the cathodal well. Two concentrations of antigen were used (undiluted and at a dilution of 8-16) to ensure maximum sensitivity. Immediately after filling the wells the trays were subjected to electrophoresis at $40 \mathrm{~mA}$ for $30 \mathrm{~min}$. The foam wicks were soaked in barbitone-sodium barbitone buffer $p \mathrm{H}$ $8 \cdot 6$, ionic strength $0 \cdot 1$. Results were read immediately against indirect light, and again after being flooded overnight with $0.5 \%$ citrate. Citrate treatment was found to enhance some positive results and to clarify doubtful results.

Immunodiffusion (ID). This was performed in the same trays as used for CIE tests, but with $20 \mathrm{ml}$ of $1 \%$ Ionagar no. 2 (Oxoid) dissolved in half-strength McIlvaine's citric acid phosphate buffer $p \mathrm{H} \mathrm{7.0}(0.05 \mathrm{M}$ citric acid $18 \mathrm{ml}, 0.1 \mathrm{M}$ disodium hydrogen phosphate $82 \mathrm{ml})$ containing $0.1 \%$ sodium azide as preservative. Well patterns were cut with a central well of 6-mm diameter surrounded by six peripheral walls of 3-mm diameter at a distance of $8 \mathrm{~mm}$, centre-to-centre, from the central well. Serum was placed in the central well and dilutions of antigen in the peripheral wells. The trays were placed in a moist chamber at $37^{\circ} \mathrm{C}$ for $2-3$ days before being examined against indirect lighting.

Fluorescent antibody test (FA). Slides were allowed to thaw at room temperature before use. Most of the control sera were reactive at dilutions of up to 20, so we used a dilution of 100 , at which the endocarditis sera gave strongly positive results. One drop of this 100 -fold dilution of serum in Coon's buffer $p \mathrm{H} \mathrm{7.2}$ was placed on each spot of the slide. After incubation for $30 \mathrm{~min}$. in a moist chamber at room temperature, the slides were washed in Coon's buffer for $30 \mathrm{~min}$. One drop of FITC-labelled anti-human globulin (Burroughs Wellcome) diluted to its optimal dilution (as determined by titration of each batch, usually 1 in 40-80) was placed on each spot and the slides incubated at room temperature for $30 \mathrm{~min}$. followed by washing in Coon's buffer for $30 \mathrm{~min}$. The slides were mounted in buffered glycerol and examined with a Reichert Zetopan Microscope with an HBO 200 Mercury Vapour lamp.

Agglutination test (AGG). Agglutination tests were performed in wells of disposable plastic trays (Stayne tray A) with $0.2 \mathrm{ml}$ of doubling dilutions in the range 1 in 20-80 of serum in saline. To each well, $0.02 \mathrm{ml}$ of $2 \%$ candida suspension was added. The trays were mixed by rotation for $30 \mathrm{~min}$. and incubated at $37^{\circ} \mathrm{C}$ overnight in a moist chamber, The cells were gently resuspended before reading for agglutination against a dark background.

Known positive and negative control sera were included in each batch of tests.

\section{RESULTS}

In the initial stages of the study, sera were screened by the CIE test only. This procedure gave a high proportion of positive, weakly positive and doubtful results. To clarify the significance of these results, other tests (ID, FA, AGG) were later introduced.

The majority of sera that gave positive reactions in the CIE test reacted against only one dilution of antigen. Treatment with $0.5 \%$ sodium citrate was found to enhance the clarity of the reaction with the majority of positive and weakly reactive sera. Most of the doubtful reactions disappeared after citrate treatment or upon re-testing.

The results with 1010 sera examined by at least one of the techniques are 


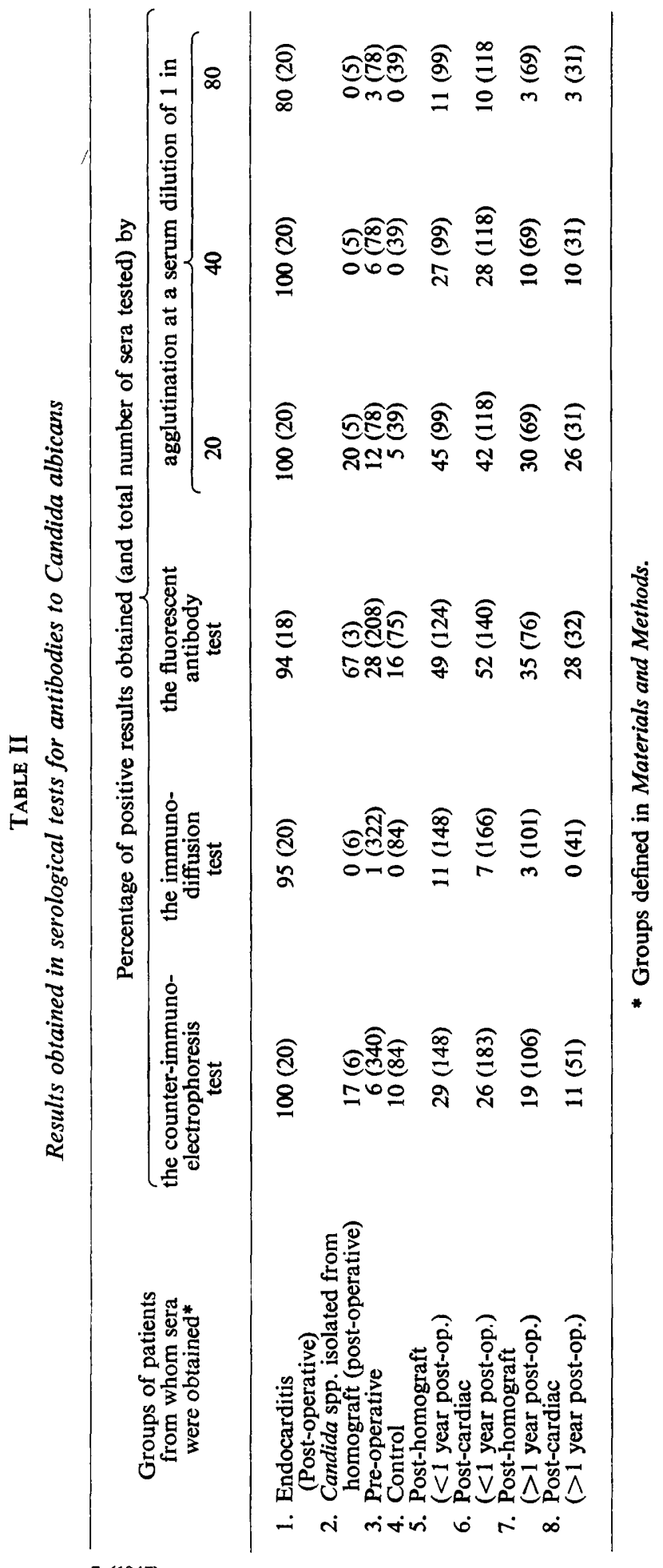


shown in table II. The sera can be divided into four groups: those from patients with endocarditis (see also table I) which gave consistently positive results in all four tests; those from pre-operative and control patients which show no significant difference and have, therefore, been combined for further analysis; those collected less than 1 year after cardiac surgery; and those collected more than 1 year after operation. No significant difference was found between results with sera from patients with homograft valve replacement and results with sera from other cardiac-surgical patients. The frequency of positive reactions was higher in the sera collected less than 1 year after operation than in those collected more than 1 year after operation. The small group of patients whose homograft tissue subsequently yielded Candida spp. did not show any significant increase in candida antibodies.

All of the endocarditis sera gave positive reactions in the CIE test (see also table I), whereas only $8 \%$ of pre-operative controls were positive. Of the postoperative sera collected at less than 1 year, $27 \%$ were positive and of those collected more than 1 year after operation, $18 \%$ were positive. In the ID test, $95 \%$ of the endocarditis sera were positive whereas less than $1 \%$ of preoperative and control sera, $9 \%$ of sera collected less than 1 year after operation, and $2 \%$ of those collected more than 1 year after operation were positive. In the FA test, $94 \%$ of the endocarditis sera, $25 \%$ of the pre-operative and control sera, $51 \%$ of those collected at less than 1 year, and $33 \%$ of those collected more than 1 year after operation, were positive. In the AGG test at a dilution of 1 in $20,100 \%$ of the endocarditis sera, $15 \%$ of pre-operative and control sera, $44 \%$ of those collected at less than 1 year, and $29 \%$ of those collected at more than 1 year after operation, were positive. At a dilution of 40 the figures are $100 \%$, $5 \%, 28 \%$, and $10 \%$ respectively.

The results with 360 sera examined by all four methods are given in table III. All of the endocarditis sera were positive in the CIE test and at least two other tests, and $87 \%$ of these sera were positive in all four tests. Of the postoperative sera, $33 \%$ of those collected less than 1 year after surgery and $19 \%$ of those collected more than 1 year after surgery were positive in three or more tests. Only $7 \%$ of the pre-operative and control sera were positive in three or more tests. Of 84 sera giving positive reactions in only one test, $52 \%$ were positive in the FA test only, 38\% in CIE only, $8 \%$ in the AGG test only, and $1 \%$ were positive in the ID test only.

\section{Discussion}

Studies of humoral antibodies to $C$. albicans by the four methods described indicate that there is a significant increase of these antibodies after operations on the heart in a proportion of patients, even in the absence of clinical evidence of endocarditis. There was no apparent difference in post-operative antibody levels of patients with homograft valve replacement in comparison with patients who received other types of cardiac surgery, although it should be noted that all of our six cases of candida endocarditis followed homograft valve replacements. The six cases of endocarditis caused by Candida spp. reported by Murray 


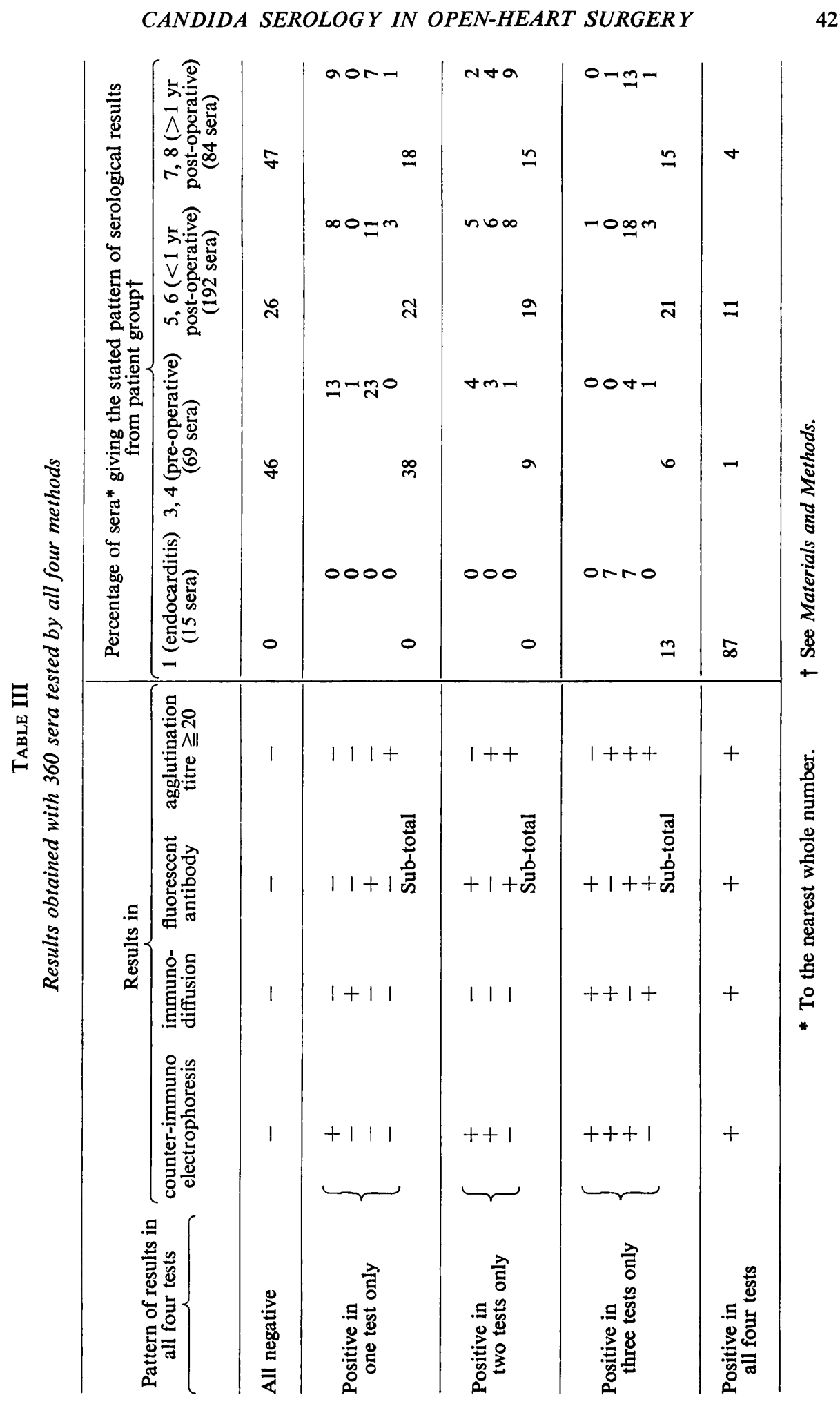


et al. (1969) also followed homografts, but in two of the three cases of candida endocarditis reported by Record et al. (1971) valve replacement was by prosthesis.

In the post-operative sera, antibodies were most often found in those obobtained less than 1 year after surgery. This may indicate a transient fungaemia in the immediate post-operative period or a general increase in the numbers of Candida spp. during post-operative chemotherapy, which all patients received. Murray et al. found no evidence that heavy proliferation of Candida spp. in the respiratory or alimentary tract was the cause of the increased antibody levels in post-operative sera. However, Krause, Matheis and Wulf (1969) demonstrated that the presence of large numbers of $C$. albicans in the alimentary tract could lead to a transient fungaemia.

Homograft valves were sterilised by a modification of the technique described by Yacoub and Kittle (1970). Strips of aortic wall from the same cadaver and sterilised in the same manner as the homograft valve were tested for sterility for periods of up to 3 months. In the six cases in which Candida spp. were isolated from these cultures, including endocarditis Case 6, the organism was not isolated until several days to 3 months after operation. The results obtained do not suggest that infected homografts are a major source of increased antibody production, and it seems likely that small numbers of Candida in a homograft can normally be tolerated by the patient.

The time-lapse between operation and symptoms of endocarditis was 6 weeks to 3 years. The cases studied by Murray et al. (1969) had a range of 5 weeks to 18 months. Endocarditis Case 5 is interesting, in that symptoms of endocarditis did not appear for more than 2 years although antibodies were present 19 days after operation. All of the post-operative sera examined, other than those in the six cases of endocarditis, were from patients without clinical evidence of endocarditis. The decreasing frequency of positive reactions with sera obtained more than 1 year after operation suggests that, if a transient fungaemia is the cause of increased antibody production, this does not necessarily result in clinical endocarditis. However, the results indicate that testing for antibodies, as an indication of possible later appearance of candida endocarditis, should be continued for at least 3 years after operation.

Blood cultures from our patients with endocarditis were often only intermittently positive and frequently failed to produce growth within 1 week. In one case, repeated blood cultures were all negative after incubation for 4 weeks. There is thus an obvious need for rapid and reliable serological tests. The most rapid technique was the CIE test, which can be read within an hour of obtaining the specimen. This test, together with the AGG test, was positive with all of our endocarditis sera. The ID test was found to be highly specific, being positive in only $1 \%$ of controls, whereas the FA test was found to be positive in $25 \%$ of control sera. The results suggest that the CIE test is useful as a routine screening technique, provided that sera giving positive results with this test are subjected to further testing by other techniques. A positive result in three or more tests should be considered as an indication for thorough investigation for endocarditis and follow-up. 


\section{SUMMARY}

Sera from patients who had undergone open-heart surgery were examined for antibodies to Candida albicans by counter-immunoelectrophoresis, immunodiffusion, immunofluorescence, and agglutination tests. No single test gave an absolute indication of systemic infection or endocarditis, although the immunodiffusion procedure was the most specific test. A counter-immunoelectrophoresis test is recommended as a screening procedure, provided that positive results are confirmed by other techniques.

Postive serological findings in the absence of clinical endocarditis is probably due to transient fungaemia but should alert the clinician to the possibility that endocarditis may develop later. Serological monitoring should be continued for at least 3 years after cardiac surgery.

We wish to thank Mr A. J. Merrick for much valuable help and advice, the Haematology Department for collection of sera, the Bacteriology Department for technical assistance, and Mrs S. Miller for typing the manuscript.

\section{REFERENCES}

Hasenclever, H. F. AND Mrtchell, W. O. 1961. Antigenic studies of Candida. I. Observations of two antigenic groups in Candida albicans. J. Bact., 82, 570.

Hellwege, H. H., Fischer, K. AND BläKeR, F. 1972. Diagnostic value of candida precipitins. Lancet, $2,386$.

Kay, J. H., Bernstein, S., Tsuji, H. K., Redington, J. V., Milgram, M. and Brem, T. 1968. Surgical treatment of candida endocarditis. J. Am. med. Ass., 203, 621.

Krause, W., Matheis, H. AND WULF, K. 1969. Fungaemia and funguria after oral administration of Candida albicans. Lancet, 1, 598.

LEHNER, T. 1966. Immunofluorescence study of Candida albicans in candidiasis, carriers and controls. J. Path Bact., 91, 97.

Murray, I. G., BuCKLey, H. R. AND Turner, G. C. 1969. Serological evidence of candida infection after open-heart surgery. J. med. Microbiol., 2, 463.

O'Neill, P. AND Johnson, G. D. 1970. Multispot immunofluorescence: a simple semiautomatic method of processing large numbers of tests. J. clin. Path., 23, 185.

Record, C. O., Skinner, J. M., Sleight, P. AND Speller, D. C. E. 1971. Candida endocarditis treated with 5-fluorocytosine. Br. med.J., 1, 262.

Remington, J. S., Gaines, J. D. and Gilmer, M. A. 1972. Demonstration of candida precipitins in human sera by counterimmunoelectrophoresis. Lancet, 1, 413.

Stallybrass, F. C. 1964. Candida precipitins. J. Path. Bact., 87, 89.

WINNER, H. I. 1955. A study of Candida albicans agglutinins in human sera. J. Hyg., Camb., $53,509$.

YACOUB, M. AND KitTLE, C. F. 1970. Sterilization of valve homografts by antibiotic solutions. Circulation, 41 and 42, Suppl., 29. 